\title{
Research on Recognition and Correction of Power System Load Singular Data Based on Wavelet Analysis
}

\author{
Chao Hong ${ }^{1}$ and Xiangshu $\mathrm{Ye}^{2}$
}

(Jingdezhen Ceramic University, Jingdezhen, Jiangxi 333403)

\begin{abstract}
Traditional singular data identification and correction methods process data roughly and cannot accurately deal with the shortcomings of singular data, this paper proposes a singular data identification and correction method based on wavelet analysis, which uses the localization properties of wavelet analysis in terms of time domain and frequency domain with the "micro" features of signals. First of all, wavelet analysis is conducted to extract the high frequency component signal as well as characterization of random noise, combined with probability statistical method to analyze the high frequency component signals, determine the occurrence time of singular data and finally eliminate singular data. The linear interpolation method is used to supplement the correction. A large number of examples show that the method is correct and effective.
\end{abstract}

Keywords: Singular data; Wavelet analysis; Singularity detection; Load forecasting; Power system

\section{一种基于小波分析的电力系统负荷奇异数据识别}

\section{及修正方法的研究}

\author{
洪超 $^{1}$, 叶向舒 ${ }^{2}$ \\ (景德镇陶瓷大学 江西省 景德镇 333000 )
}

摘要: 针对传统的奇异数据识别和修正方法对数据处理粗精, 难以精确处理奇异数据的缺点, 本文提出了一种基于小波 分析的奇异数据识别及修正方法，该方法利用小波分析在时域和频域同时具有良好的局部化性质，对信号具有 “显微”的 特性, 首先对信号进行小波分析, 提取出表征随机喿声的高频分量, 再结合概率统计的思想对高频分量信号进行分析, 确定 奇异数据的发生时刻, 最后剔除奇异数据, 采用线性插值方法进行补充修正。大量计算实例表明了该方法的正确性和有效性。

关键词: 奇异数据; 小波分析; 奇异性检测; 负荷预测; 电力系统

中图分类号: F416.61 文摘标识码: A

引言

电力负荷预测是依赖于大量历史资料及相关因素资料的被动型预测，预报者占有资料的翔实可靠程 度, 对基于任何方法的预报结果都会有很大影响。目前, 在我国各级电力调度中心, 历史负荷数据采集系 统多为 SCADA系统。在电力系统实际运行时, 数据采集系统中的量测、记录、转换、传输过程的任意环节 都可能引起故障而导致观测数据的反常态势, 以致与大多数观测值与实际值不一致; 另一方面, 当数据采 集系统正常, 由于特殊事件 ( 如切负荷停电, 线路检修停电, 大用户、大事件冲击等) 引起负荷的异常变 化, 也会导致观测数据的违背常规。所有这些非正常的观测数据统称为奇异数据 ${ }^{[1]}$ 。如果这些奇异数据得 不到有效的校正, 很有可能将误导负荷预测模型的建立, 影响预测结果的精确度和可靠性。

在实际的工程应用中, 常用到的奇异数据识别及修正方法有以下几种: 经验修正法、曲线置换法, 纵 向对比法、概率统计法等等, 尽管这些方法都非常简单, 且具有很强的实用性, 但是它们存在一个共同的 缺点, 就是数据处理较为粗粘, 不能精确的处理奇异数据, 很难保证负荷预测模型对历史数据的要求。为 了能够更好的处理奇异数据, 本文提出了一种新的基于小波分析的奇异数据识别及修正方法, 该方法不同 
于小波变换的模极大值理论 ${ }^{[2]}{ }^{[3]}$, 而是利用小波的重构技术, 提取出表征随机噪声的高频分量, 结合概率 统计的思想对高频分量信号进行统计分析, 进而确定奇异数据发生的时刻, 最后采用线性插值的方式对信 号进行修正。计算实例表明了该方法是非常有效的。

\section{1. 小波分析理论简介}

小波分析是近10年来兴起的一种新的信号分析技术，是针对传统的Fourier分析与其后的加窗Fourier 分析的不足提出的一种方法 ${ }^{[4]}$, 小波分析能够分析信号的局部特点, 尤其适合分析一些存在突变, 不连续 等的复杂信号，所以小波分析技术一经提出就在各个领域得到了广泛应用。

连续小波变换公式如下 ${ }^{[5]}$ :

$$
T^{w a v}(a, b)=|a|^{-1 / 2} \int_{-\infty}^{+\infty} f(t) \overline{\Psi_{a, b}(t)} d t
$$

式中 $a$ 为尺度因子, $b$ 为定位参数。

其中函数 $\Psi_{a, b}(t)$ 称为小波函数或者母小波,

$\Psi_{a, b}(t)=\frac{1}{\sqrt{|a|}} \Psi\left(\frac{t-b}{a}\right)$

且它必须满足容许性条件:

$$
C_{\psi}=\int_{-\infty}^{+\infty} \frac{\left.\hat{\psi(\omega)}\right|^{2}}{|\omega|} d \omega<+\infty
$$

此时可以由小波的反演公式对信号进行重构, 反演公式如下 ${ }^{[6]}$ :

$$
f(t)=\frac{1}{C_{\psi}} \int_{-\infty}^{+\infty} \int_{-\infty}^{+\infty} a^{-2} T^{w a v}(a, b) \psi_{a, b}(t) d a d b
$$

小波变换是一种工具，它把数据、函数、或算子分割成不同频率的成分，然后再用分解的方法去研究 对应尺度下的更细的成分, 当改变尺度参数 $\alpha$ 的值, $\Psi_{a, 0}(t)=|a|^{-1 / 2} \psi(t / a)$ 覆盖不同的频率范围, 其中, $|a|$ 的大值对应于低频或大尺度的 $\Psi_{a, 0}$, 而 $|a|$ 的小值对应于高频或者小尺度的 $\Psi_{a, 0}$, 改变参数 $\mathrm{b}$ 时相当于移 动时窗中心, 每一个 $\Psi_{a, b}(t)$ 都被定位在 $\mathrm{t}=\mathrm{b}$ 附近 ${ }^{[7]}$ 。

小波函数 $\Psi_{a, b}(t)$ 经过整数缩放和整节点平移生成的时间一频率空间的函数族, 构成了离散小波, 如 果取对尺度参数进行二进制离散 (即: $a=1 / 2^{j}, j \in Z$ ), 而平移参数保持原来的离散变化, 则这种 小波变换被称为二进小波变换。这种二进变换在实际应用中使用较多。

小波变换技术本质上与加窗傅立叶变换一样是一种时一频定位技术，只不过加窗傅立叶变换是采用正 弦波分析信号的成分, 且窗框不变, 而小波变换则采用的是能够随着尺度的变化而自适应伸缩的小波来对 信号进行分析的, 正因为这些不同, 小波对奇异信号的检测具有其他分析方法没有的优点, 从而奠定了它 在工程界的地位。 


\section{2. 基于小波分析的奇异数据识别及修正方法}

\section{1 小波除噪原理}

由于不同类型的负荷曲线存在高峰和低谷及随机噪声的影响而出现的正常波动现象，但是它们并没有 超出常规的数值, 在信号的奇异性检测过程中, 这些正常的波动也可能导致对信号进行小波分析时产生误 判, 认为是奇异点, 所以为了尽量避免由于信号的正常波动所引起的奇异性误判, 应该在奇异信号辨别的 过程中首先对信号进行除噪, 即: 设置一个坎值分离出正常波动导致的不良数据, 从而提高奇异数据识别 的准确率与效率。

坎值设置: 大量的研究表明, 随机噪声信号一般服从正态分布 ${ }^{[8]}$, 所以本文认为, 负荷数据的随机噪 声同样服从正态分布, 进而对信号的高频分量进行正态分布的参数区间估计, 得到分布参数 $\mu, \sigma$ 和相应 置信度的置信区间。

正态分布概率密度函数:

$$
f(x)=\frac{1}{\sqrt{2 \pi} \sigma} e^{\frac{-(x-u)^{2}}{2 \sigma^{2}}}
$$

参数估计，对 $\mu$ 进行的置信度为 0.9 的置信区间估计，即满足：

$$
P(\theta<\mu<\bar{\theta})=0.9
$$

式中 $\theta 、 \bar{\theta}$ 分别表示置信区间的下限和上限。

区间参数估计置信度的反应了区间估计的可靠性，置信度越大表明估计参数在估计区间内可靠性越 高, 即均值在此区间内的可能性越大。根据区间参数估计的实际意义, 本文认为参数 $\mu$ 的置信度为 0.9 的 置信区间内的值即为正常的波动, 区间外波动较大的值认为是非正常波动, 即认为是奇异数据, 从而确定 原始信号奇异数据发生的位置。

\section{2 方法的整体思想}

该方法利用小波分析在时域和频域同时具有良好的局部化性质，且对信号具有 “显微” 的特性，对奇 异信号的识别具有独特优点, 首先采用小波分析对信号进行分解, 由于奇异信号与一些正常的噪声信号都 体现在信号的高频部分, 所以利用小波分析再对信号的高频部分进行重构, 再结合本文提出的小波除噪原 理, 从重构的高频时域信息中来确定奇异信号发生的时刻, 将奇异信号提取出来, 最后采用线性插值的方 式对奇异数据进行修正。

实践表明, DB1 小波对信号的不连续及突变识别能力较强, 而且具有广泛的适用性, 本文采用离散二 进制DB1小波作为母小波对信号进行分析。

\section{3 方法的实现步骤}

1) 对原始信号进行小波分解, 得到各频率成分的小波系数。

2）对高频分量进行重构, 得到时域内高频分量信号D1。

3）使用本文提出的小波除噪方法对高频信号进行除噪并确定奇异数据发生时刻。

4) 剔除奇异数据, 采用线性插值的方式进行补充修正, 如果某个区域都为奇异数据, 即奇异数据连 
续出现时, 则插值公式中的 $y_{k-1}, y_{k+1}$ 取区域左右外的第一个值。

线性插值公式:

$$
L\left(t_{k}\right)=\frac{t_{k}-t_{k+1}}{t_{k-1}-t_{k+1}} y_{k-1}+\frac{t_{k}-t_{k-1}}{t_{k+1}-t_{k-1}} y_{k+1}
$$

式中: $L\left(t_{k}\right)$ 表示所插的值。

$t_{k}$ 表示插值时刻。

$y_{k+1}$ 表示 $t_{k+1}$ 时刻的历史数据。

\section{3. 实例分析}

对某电网某日电力负荷奇异数据的识别及修正, Scada系统采集为 5 分钟一点, 即：一日 288 点。 下图为实际负荷历史数据:

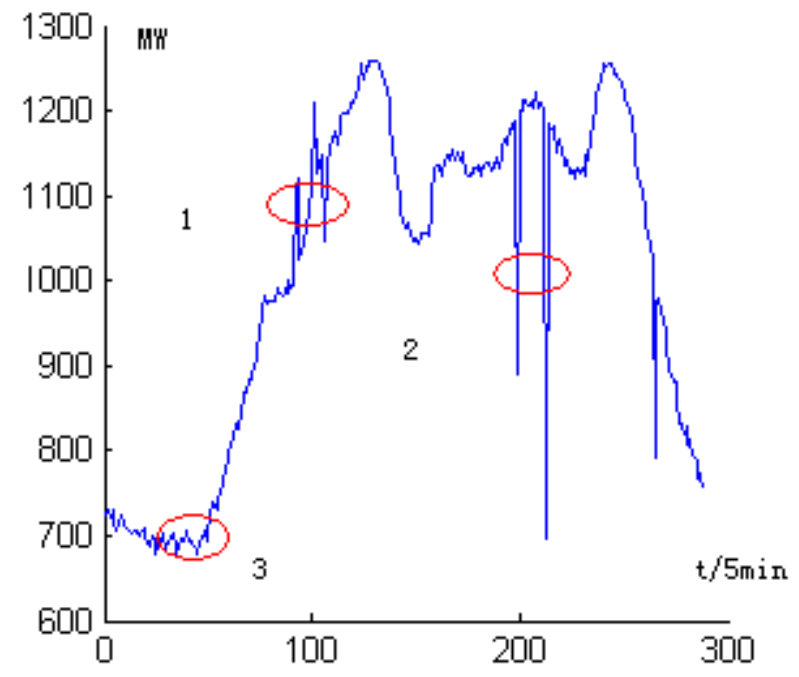

图 1 历史数据

从图中可以看出, 历史数据中既包含区域 $1 、 2$ 这种波动较大的数据, 又有象区域 3 这样的毛刺部分, 这些奇异值并不连续, 在实际工程应用中, 为了能够给负荷预测等软件提供一个良好的数据基础, 这些数 据一般都得进行修正，包括去除毛刺部分和修正波动较大的奇异数据。

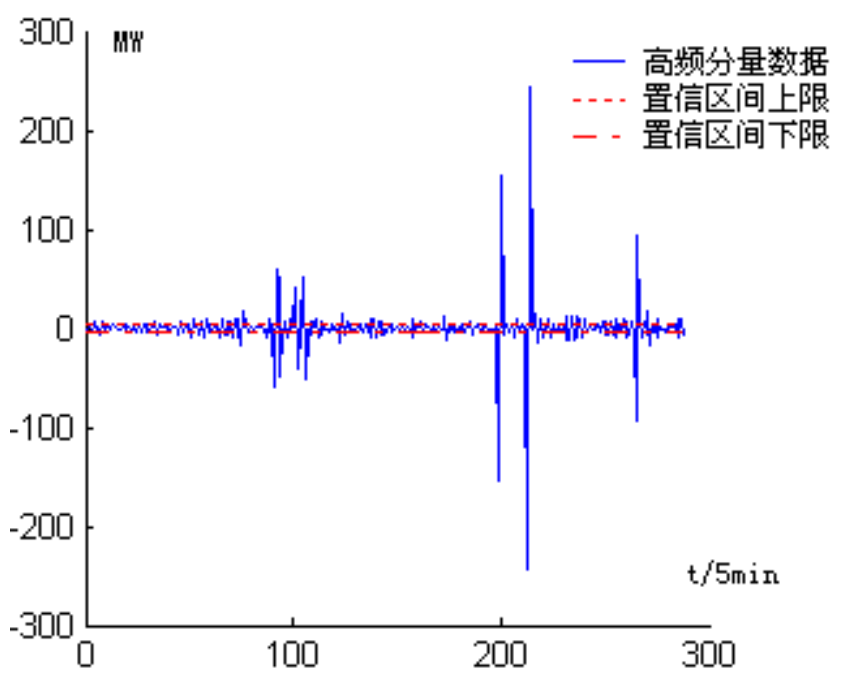

图 2 一尺度下小波分解 D1 分量 
通过对历史数据在一尺度下的小波变换, 可以发现, 小波对奇异数据的是非常敏感的, 这样就可以充 分的发现奇异数据，也体现了小波分析的优越性。

通过对高频分量的参数区间估计，得到 $\mu=0$, 且置信度为 0.9 的置信区间为 $(-2.6539,2.6539)$, $\sigma=27.293$ 。

将区域内的波动分量认为是正常的波动，应该当成一般的毛刺去除。去除毛刺后还剩下的数据部分即 认为是波动较大的奇异数据, 下图为去除毛刺后剩下的部分, 即奇异数据。

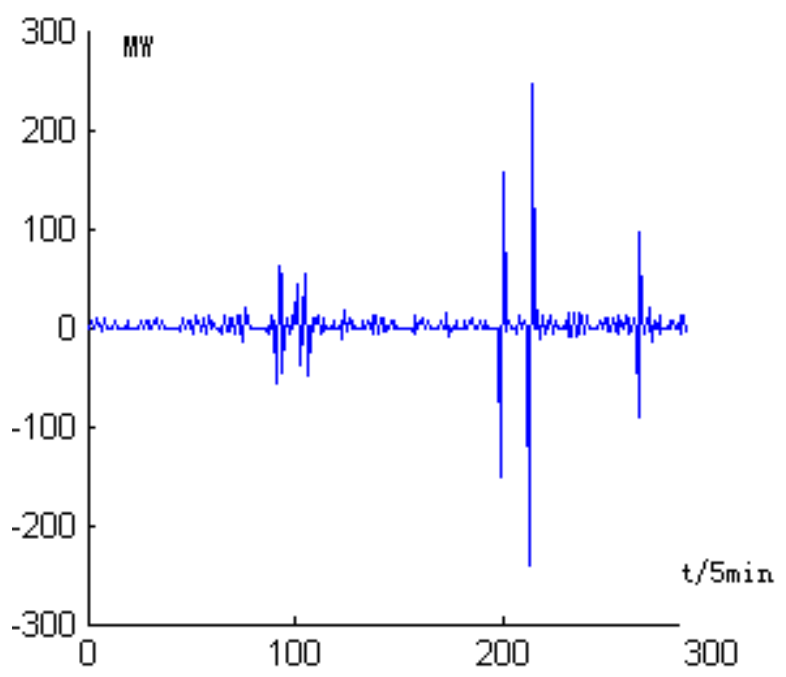

图 3 奇异数据

图3中表明了奇异数据高频分量值及发生的时刻, 其中毛刺部分全部置零。通过高频分量的奇异数据, 确定历史数据中存在的奇异数据位置, 将其剔除, 采用线性插值方式进行修正, 修正后如下图:

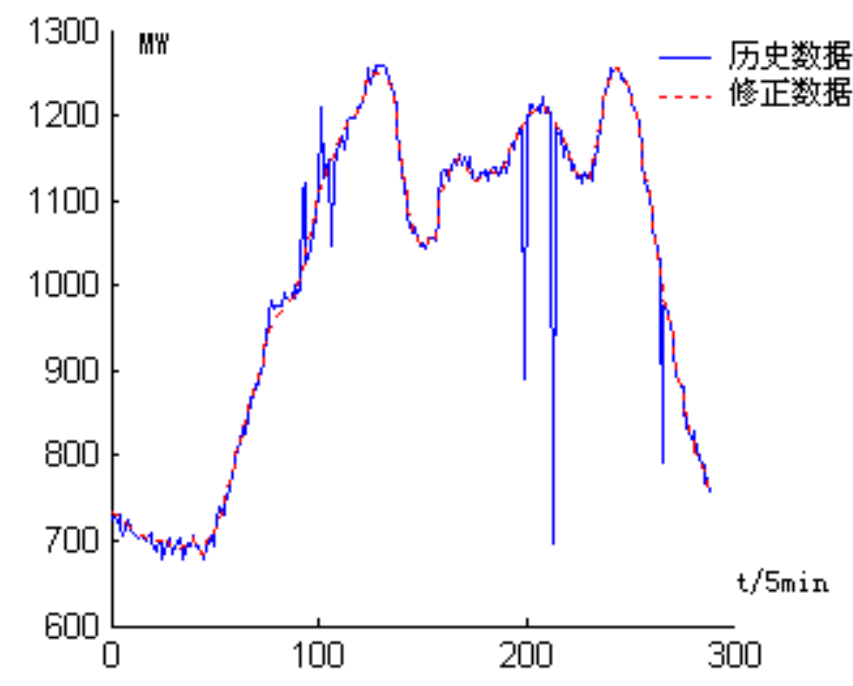

图 4 修正后与历史数据比较

从图中可以看出该方法对奇异数据的修正效果很好。

\section{4. 结论}

本文提出的基于小波分析奇异数据识别及修正方法可以有效的检测出由于信号中不良数据所导致的 奇异点, 并对其进行修正, 该方法简单易懂, 计算方便, 通用性强, 可以为电力系统负荷预测、EMS等高 级应用软件提供良好的数据基础。 
通过对电力系统负荷实际数据的分析检验，证明了该方法的正确性和有效性。

\section{参考文献:}

[1] 田增尧, 张明理, 赵瑞。短期电力负荷预报中异常负荷数据的识别和修正 $[\mathrm{J}]$ 。吉林电力，2004, 第六期；21-23。

[2] 李慧，杨明皓. 小波分析在电力系统不良数据辨识中的应用 [J].继电器，2005，33(3):10-14.

[3] 李天云, 胡屏, 马景兰. 小波奇异性检测理论在电力系统负荷特性分析中的应用 [J]. 东北电力学院学报, 1998 , $18(4): 14-22$.

[4] 陈祥训. 对几个小波基本概念的理解 [J]. 电力系统自动化，2004，28(1):1-6.

[5] 邰能灵, 候志俭, 李涛, 蒋传文, 宋畑. 基于小波分析的电力系统短期负荷预测方法 [J]. 中国电机工程学报, 2003, $23(1): 45-50$.

[6] 任震 等著. 小波分析及其在电力系统中的应用 [M]. 北京: 中国电力出版社.

[7] Ingrid Daubechies著, 李建平, 杨万年等译. 小波十讲 [M]. 北京: 国防工业出版社.

[8] 刘乐平, 段五朵. 概率论与数理统计 (第三版) [M]. 江西, 江西高校出版社.

\section{作者简介:}

洪超（1982-）男，实验师，景德镇陶瓷大学，研究方向为电力系统空间负荷预测。

叶向舒 (1981-) 女, 讲师, 景德镇陶瓷大学, 研究方向: 英美文学及翻译, 英美文化。

\section{References:}

[1] Tian Zengyao, Zhang Mingli, Zhao Rui Identification and correction of abnormal load data in short - term load forecasting [J]. Jilin Electric Power, 2004, sixth period; 21-23.

[2] LI Hui, YANG Ming-hao. Application of Wavelet Analysis in Bad Data Identification of Power System [J]. Journal of Electronics \& Technology, 2005, 33 (3): 10-14.

[3] Li Tianyun, Hu Ping, Ma Jinglan. Application of wavelet singularity detection theory in load characteristics analysis of power system [J]. Journal of Northeast Dianli University, 1998, 18 (): 14-22.

[4] Chen Xiangxun. Understanding of Several Fundamental Concepts of Wavelet [J]. Automation of Electric Power Systems, 2004, 28 (1): 1-6.

[5] TAI Neng-ling, SHI Zhi-jian, LI Tao, JIANG Chuan-wen, SONG Jiong. Remote energy load forecasting method based on wavelet analysis [J]. Proceeding of the CSEE, 2003, 23 (1): 45-50.

[6] FENG Zhen et al. Wavelet Analysis and Its Application in Power System [M]. Beijing: China Electric Power Press. 